SIAM REviEw, originally submitted on July 7, 2006, revised versions submitted on February 9, 2007 and on June 20, 2007

\title{
HOW TO TRANSFORM ONE CONVEX FUNCTION CONTINUOUSLY INTO ANOTHER*
}

\author{
HEINZ H. BAUSCHKE ${ }^{\dagger}$, YVES LUCET ${ }^{\ddagger}$, AND MICHAEL TRIENIS ${ }^{\S}$
}

\begin{abstract}
The proximal average operator provides a parametric family of convex functions that continuously transform one convex function into another even when the domains of the two functions do not intersect. We prove that the proximal average operator is a homotopy with respect to the epi-topology, study its properties, and present several explicit formulas for specific classes of functions. The parametric family inherits desirable properties such as differentiability and strict convexity from the given functions.

The results illustrate the powerful tools available in convex and variational analysis from both a theoretical and a computational point of view.
\end{abstract}

Key words. Attouch-Wets topology, continuous transformation, convex function, deformation, epi-convergence, epi-topology, Fenchel conjugate, homotopy, proximal average, proximal mapping

AMS subject classifications. 90C25, 26A51, 26B25, 26E60, 46B03, 90-01

\section{PII. XXXXX}

\section{Introduction.}

The idea of potentially connecting mathematical objects in a continuous fashion is fundamental: in geometry, points might be connected through paths; in approximation theory, data points might be connected by some interpolant; in topology, functions might be transformed into each other via homotopies. For instance, consider the two functions

$$
f_{0}(x)=x+2 \quad \text { and } \quad f_{1}(x)=x^{2}
$$

How can we transform $f_{0}$ continuously into $f_{1}$ ? Perhaps the most natural approach is to use the pointwise arithmetic average

$$
(1-\lambda) f_{0}+\lambda f_{1}
$$

where $\lambda \in[0,1]$. This transformation has very nice properties: the intermediate functions $(1-\lambda) f_{0}+\lambda f_{1}$ are all continuous, and for every $x \in \mathbb{R}$, the mapping $\lambda \mapsto f_{\lambda}(x)$ is also continuous. See Fig. 1.1(a) for a visualization. This approach works very well as long as the two given functions $f_{0}$ and $f_{1}$ are continuous and they share the same domain; however, it strikingly breaks down if $f_{0}$ and $f_{1}$ have disjoint

*Versions submitted on July 7, 2006; on February 9, 2007; and on June 20, 2007.

http://www.siam.org/journals/sirev/x-x/XXXX.html

$\dagger$ Mathematics, Irving K. Barber School, University of British Columbia Okanagan, Kelowna, British Columbia V1V 1V7, Canada (heinz.bauschke@ubc.ca). Research partially supported by the Natural Sciences and Engineering Research Council of Canada and by the Canada Research Chair Program.

$\ddagger$ Computer Science, Irving K. Barber School, University of British Columbia Okanagan, Kelowna, British Columbia V1V 1V7, Canada (yves.lucet@ubc.ca). Research partially supported by the Natural Sciences and Engineering Research Council of Canada.

$\S$ Computer Science, Irving K. Barber School, University of British Columbia Okanagan, Kelowna, British Columbia V1V 1V7, Canada (mjtrieni@interchange.ubc.ca). Research partially supported by the Pacific Institute for the Mathematical Sciences. 


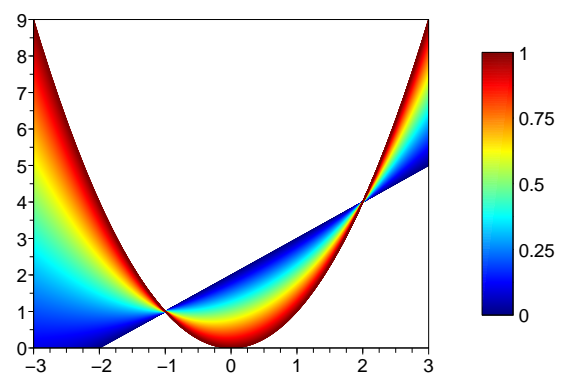

(a) From $f_{0}$ to $f_{1}$ : Arithmetic Average

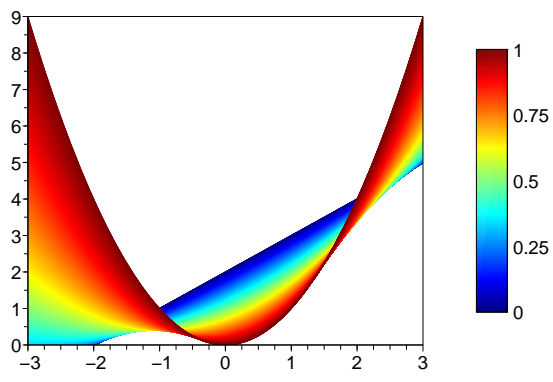

(b) From $f_{0}$ to $f_{1}$ : Proximal Average

FIG. 1.1. Averages of the linear function $f_{0}(x)=x+2$ and the quadratic function $f_{1}(x)=x^{2}$.

domains because then the functions values cannot be averaged arithmetically. At this point, it is not at all clear how one could transform

$$
f_{0}(x)=-\ln (-x) \quad \text { and } \quad f_{1}(x)=f_{0}(-x)=-\ln (x)
$$

continuously into each other because the domains of these functions are, respectively, the disjoint intervals $]-\infty, 0[$ and $] 0,+\infty[$.

Quite surprisingly, convex analysis will pave the way to transform these two functions (and many other convex functions) into each other. The average which provides this transformation is called the proximal average and denoted by

$$
\mathcal{P}\left(f_{0}, \lambda, f_{1}\right),
$$

where $\lambda \in[0,1]$.

Although we are not yet ready to describe its inner workings, let us nonetheless provide two visualizations of the proximal average. Fig. 1.1(b) illustrates the proximal average for the two functions $f_{0}$ and $f_{1}$ from (1.1), while Fig. 1.2 illustrates the proximal average for the two logarithms defined in (1.2).

As will become clear in the following sections, the proximal average has various desirable properties that the arithmetic average generally lacks. For instance, if one of the given functions is differentiable, then so is the proximal average. Similarly, if one of the given functions is defined on the entire real line, then so is the proximal average.

The goal of this note is to introduce and study the proximal average. This transformation requires some basic convex calculus and it is thus accessible to students taking advanced undergraduate or beginning graduate courses in convex optimization and analysis. The material presented here provides a nice motivation for convex and variational analysis. It can also be used as a new topic in a corresponding course and - as we have not seen the main results elsewhere - possibly as a starting point for further research.

The remainder of the paper is organized as follows. In Section 2, we set the stage by reviewing notions from convex optimization and analysis. The proximal average is defined in terms of Fenchel conjugates, which are introduced in Section 3. We then present in Section 4 the formula for the proximal average as well as basic properties and examples. Section 5 introduces epi-convergence of functions. The associated topology, 


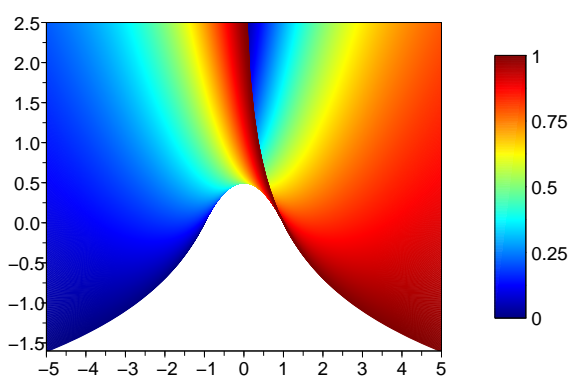

FIG. 1.2. The proximal average of $f_{0}$ and $f_{1}$, as defined in (1.2). Note that $\operatorname{dom} f_{0} \cap \operatorname{dom} f_{1}=\varnothing$ - which shows that the arithmetic average is of no use in such settings - and that for $\lambda \in] 0,1[$, the proximal average $\mathcal{P}\left(f_{0}, \lambda, f_{1}\right)$ has full domain.

the epi-topology, turns out to be exactly the right notion to establish continuity of the proximal average operator (Theorem 5.4). As demonstrated in Section 6, the proximal average inherits good properties - such as smoothness or strict convexity - from the functions it averages. As an application, we present a renorming result (Theorem 6.5). Additional examples of proximal averages are provided in Section 7, which can be viewed as a source of exercises. Some more technical results and proofs are relegated to the Appendices.

\section{Convex Optimization: Basic Set Up.}

From now on, we assume that

$$
X=\mathbb{R}^{d} \text { with inner product }\langle x, y\rangle=x^{T} y \text { and norm }\|x\|=\sqrt{\langle x, x\rangle} .
$$

The most basic constrained optimization problem is to

$$
\text { minimize } f \text { over } C \text {, }
$$

where $f$ is called the objective function and $C$ is the constraint set. The indicator function corresponding to $C$ is

$$
\left.\left.\iota_{C}: X \rightarrow\right]-\infty,+\infty\right]=\mathbb{R} \cup\{+\infty\}: x \mapsto \begin{cases}0, & \text { if } x \in C ; \\ +\infty, & \text { otherwise }\end{cases}
$$

Working with $+\infty$ and indicator functions allows us to express (2.1) as the equivalent unconstrained optimization problem

$$
\text { minimize } f+\iota_{C} \text { over } X \text {. }
$$

This is a common theme in modern convex analysis and optimization; see, e.g., [8, 9, 13, 20, 21]. These books also serve as reference material for notation and results not explicitly defined here. Now fix a function $f: X \rightarrow]-\infty,+\infty]$. Then $f$ is convex if

$$
(\forall x \in X)(\forall y \in X)(\forall \lambda \in] 0,1[) \quad f((1-\lambda) x+\lambda y) \leq(1-\lambda) f(x)+\lambda f(y) ;
$$

and $f$ is lower semicontinuous if

$$
x_{n} \rightarrow x \Rightarrow f(x) \leq \underline{\lim } f\left(x_{n}\right),
$$




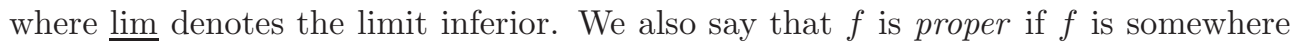
finite. A more geometric view of these three concepts rests on the notion of the epigraph of $f$, which is defined by

$$
\text { epi } f:=\{(x, r) \in X \times \mathbb{R} \mid f(x) \leq r\}
$$

and which consists of all points lying on or above the graph of $f$. Recall that a set $C$ is convex if $(1-\lambda) x+\lambda y$ belongs to $C$ whenever $x \in C, y \in C$, and $\lambda \in[0,1]$. Then one may check that $f$ is convex, lower semicontinuous, and proper if and only if the set epi $f$ is convex, closed, and nonempty, respectively. The (effective) domain of $f$ is

$$
\operatorname{dom} f:=\{x \in X \mid f(x) \in \mathbb{R}\} ;
$$

it coincides with the projection of epi $f$ onto the $X$-component. The function $f$ has full domain if $\operatorname{dom} f=X$. We shall work with the following class of functions.

$$
\mathcal{F}:=\{f: X \rightarrow]-\infty,+\infty] \mid f \text { is convex, lower semicontinuous, and proper }\} .
$$

Example 2.1. Let $C$ be a subset of $X$. Then the indicator function $\iota_{C}$ belongs to $\mathcal{F}$ if and only if the set $C$ is convex, closed, and nonempty.

The next example is the multi-dimensional analogue of the function $f_{1}$ from (1.2). It lies at the heart of interior point methods in linear optimization.

EXAMPle 2.2 (logarithmic barrier). Set $\mathbb{R}_{++}^{d}:=\left\{x \in \mathbb{R}^{d} \mid\right.$ each $\left.x_{i}>0\right\}$ and

$$
\left.\left.\mathrm{lb}: \mathbb{R}^{d} \rightarrow\right]-\infty,+\infty\right]: x \mapsto \begin{cases}-\sum_{i=1}^{d} \ln \left(x_{i}\right), & \text { if } x \in \mathbb{R}_{++}^{d} ; \\ +\infty, & \text { otherwise. }\end{cases}
$$

Then $\mathrm{lb}$ belongs to $\mathcal{F}$.

\section{Convex Optimization: Fenchel Conjugates.}

We require one more notion from convex optimization before we are able to write down the formula for the proximal average. The Fenchel conjugate of a proper function $f: X \rightarrow]-\infty,+\infty]$ is defined by

$$
\left.\left.f^{*}: X \rightarrow\right]-\infty,+\infty\right]: x^{*} \mapsto \sup _{x \in X}\left\langle x, x^{*}\right\rangle-f(x) .
$$

Let us briefly comment on a geometric interpretation. Fix $x^{*} \in X$, thought of as a "slope", and consider the family $\left(H_{s}\right)_{s \in \mathbb{R}}$ of all hyperplanes in $X \times \mathbb{R}$ with normal vector $\left(x^{*},-1\right)$, where

$$
H_{s}:=\left\{(x, r) \in X \times \mathbb{R} \mid\left\langle x, x^{*}\right\rangle-r=s\right\}=\left\{\left(x,\left\langle x, x^{*}\right\rangle-s\right) \mid x \in X\right\} .
$$

Given a fixed $s \in \mathbb{R}$, one of the following two mutually exclusive cases occurs.

Case 1: The graph of $f$ (and hence epi $f$ ) lies above or on the hyperplane $H_{s}$, i.e., $(\forall x \in \operatorname{dom} f) f(x) \geq\left\langle x, x^{*}\right\rangle-s$; equivalently,

$$
s \geq f^{*}\left(x^{*}\right) .
$$

Case 2: There exists a point $(x, f(x))$ on the graph of $f$ lying strictly below the hyperplane $H_{s}$, i.e., $f(x)<\left\langle x, x^{*}\right\rangle-s$; equivalently,

$$
s<f^{*}\left(x^{*}\right) .
$$


The two cases together imply that $f^{*}\left(x^{*}\right)$ is the minimum of all values $s$ for which the graph of $f$ lies above or on the hyperplane $H_{s}$. (If $f^{*}\left(x^{*}\right)=+\infty$, then no hyperplane $H_{s}$ lies below or on the graph of $f$.) Thus, the hyperplane $H_{f^{*}\left(x^{*}\right)}$ intersects the vertical $\{0\} \times \mathbb{R}$ axis at the largest possible height $-f^{*}\left(x^{*}\right)$. (See also [13, Chapter $\left.\mathrm{X}\right]$ for another geometric interpretation.) If $f$ is differentiable everywhere, then $f^{*}$ coincides with the classical Legendre transformation.

We now review basic results on Fenchel conjugates. The Biconjugate Theorem states that

$$
(\forall f \in \mathcal{F}) \quad f^{* *}=f .
$$

The energy function $\frac{1}{2}\|\cdot\|^{2}$ is the only self-conjugate function, i.e.,

$$
(\forall f \in \mathcal{F}) \quad f=f^{*} \quad \Leftrightarrow \quad f=\frac{1}{2}\|\cdot\|^{2} .
$$

Fenchel conjugates naturally arise in convex optimization. For example, the Fenchel-Rockafellar Duality Theorem states that given $f \in \mathcal{F}, g \in \mathcal{F}$, if $\operatorname{dom} f$ has a nonempty intersection with the interior of $\operatorname{dom} g$, then the primal optimization problem

$$
\inf _{x \in X} f(x)+g(x)
$$

has the same value as the dual optimization problem

$$
-\inf _{x^{*} \in X} f^{*}\left(-x^{*}\right)+g^{*}\left(x^{*}\right)
$$

and the latter infimum is actually attained, i.e., a minimum.

In Fourier analysis, convolution is a fundamental operation because it is dual to multiplication via Fourier transforms. In convex analysis, the counterpart to the convolution is the so-called infimal convolution, defined by

$$
f \square g: X \rightarrow[-\infty,+\infty]: x \mapsto \inf _{y \in X} f(y)+g(x-y) .
$$

Indeed, the infimal convolution is dual to addition via Fenchel conjugation in the sense that $(f \square g)^{*}=f^{*}+g^{*}$ and that $(f+g)^{*}=f^{*} \square g^{*}$ if $\operatorname{dom} f$ meets the interior of $\operatorname{dom} g$.

Let us present the conjugate of the so-called negative entropy.

EXAmple 3.1 (negative entropy). Set $\mathbb{R}_{+}^{d}:=\left\{x \in X \mid\right.$ each $\left.x_{i} \geq 0\right\}$. With the standard convention that $0 \ln (0)=0$, define

$$
\text { negent: } \left.\left.\mathbb{R}^{d} \rightarrow\right]-\infty,+\infty\right]: x \mapsto \begin{cases}\sum_{i=1}^{d} x_{i} \ln \left(x_{i}\right)-x_{i}, & \text { if } x \in \mathbb{R}_{+}^{d} ; \\ +\infty, & \text { otherwise. }\end{cases}
$$

Then negent belongs to $\mathcal{F}$ and negent ${ }^{*}=\exp$, where $\exp : \mathbb{R}^{d} \rightarrow \mathbb{R}: x \mapsto \sum_{i=1}^{d} \exp \left(x_{i}\right)$.

Proof. It is clear that negent $\in \mathcal{F}$. Let us compute its conjugate. To this end, it suffices to consider the case when $d=1$ since in the general case the supremum of each coordinate can be taken separately. Fix $y \in \mathbb{R}$. Then $f^{*}(y)=\sup _{x}(y x-(x \ln x-x))$. We supremize a concave function, so every critical point is a maximizer. Solving the critical point equation $0=\frac{d}{d x}(y x-(x \ln x-x))=y-\ln x$, we see that $x=e^{y}$ is the unique maximizer and hence $f^{*}(y)=y e^{y}-\left(e^{y} \ln \left(e^{y}\right)-e^{y}\right)=e^{y}$.

To whet the reader's appetite further, we visualize in Fig. 3.1 the proximal averages from the negative entropy to its conjugate, and from a quadratic function to an indicator function. 


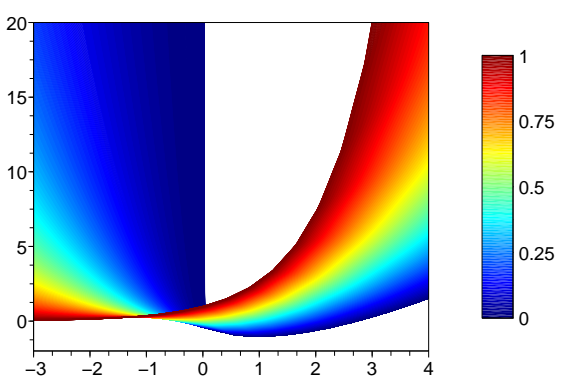

(a) From negent to exp

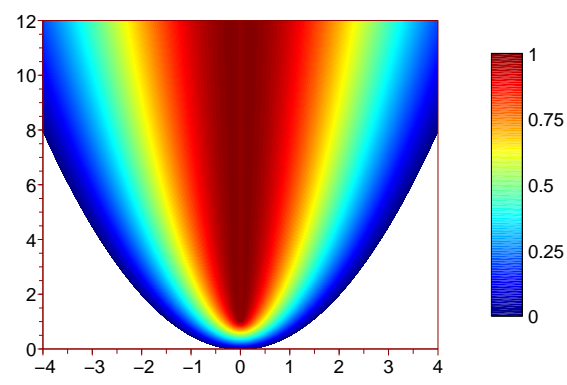

(b) From $\frac{1}{2}\|\cdot\|^{2}$ to $\iota_{\{0\}}$

FIG. 3.1. Proximal averages of two functions with different domains.

\section{Proximal Average.}

The formula for the proximal average $\mathcal{P}$ appeared, in an equivalent form, first in [4], which dealt with aspects of convergence of certain fixed point iterations. The proximal average was used there to provide an explicit constructive proof of Moreau's observation [18] that the set of all so-called proximal mappings is convex - this is the motivation for the term "proximal average".

DEFINITION 4.1 (proximal average). The proximal average operator is

$$
\begin{aligned}
\mathcal{P}: & \mathcal{F} \times[0,1] \times \mathcal{F} \rightarrow\{f \mid f: X \rightarrow[-\infty,+\infty]\} \\
\left(f_{0}, \lambda, f_{1}\right) & \mapsto\left((1-\lambda)\left(f_{0}+\frac{1}{2}\|\cdot\|^{2}\right)^{*}+\lambda\left(f_{1}+\frac{1}{2}\|\cdot\|^{2}\right)^{*}\right)^{*}-\frac{1}{2}\|\cdot\|^{2} .
\end{aligned}
$$

The next result is an immediate consequence of the definition and (3.1).

Proposition 4.2. Let $f_{0} \in \mathcal{F}$, let $f_{1} \in \mathcal{F}$, and let $\lambda \in[0,1]$. Then

$$
\mathcal{P}\left(f_{0}, 0, f_{1}\right)=f_{0}, \quad \mathcal{P}\left(f_{0}, 1, f_{1}\right)=f_{1}, \text { and } \mathcal{P}\left(f_{0}, \lambda, f_{1}\right)=\mathcal{P}\left(f_{1}, 1-\lambda, f_{0}\right) .
$$

For $\lambda \in] 0,1[$, it appears on first glance to be unlikely that the proximal average $\mathcal{P}\left(f_{0}, \lambda, f_{1}\right)$ is a convex function (since the difference between convex functions generally fails to be convex). However, $\mathcal{P}\left(f_{0}, \lambda, f_{1}\right)$ is not only convex, but its conjugate is the corresponding proximal average of the conjugates!

Theorem 4.3. Let $f_{0} \in \mathcal{F}$, let $f_{1} \in \mathcal{F}$, and let $\lambda \in[0,1]$. Then $\mathcal{P}\left(f_{0}, \lambda, f_{1}\right)$ belongs to $\mathcal{F}$, and

$$
\left(\mathcal{P}\left(f_{0}, \lambda, f_{1}\right)\right)^{*}=\mathcal{P}\left(f_{0}^{*}, \lambda, f_{1}^{*}\right)
$$

Proof. See Appendix A or [4, Theorem 6.1].

REMARK 4.4. Let $f_{0} \in \mathcal{F}$, let $f_{1} \in \mathcal{F}$, and let $\lambda \in[0,1]$. Set $f_{\lambda}:=\mathcal{P}\left(f_{0}, \lambda, f_{1}\right)$ and $\left(f^{*}\right)_{\lambda}:=\mathcal{P}\left(f_{0}^{*}, \lambda, f_{1}^{*}\right)$. Then Theorem 4.3 states that

$$
\left(f_{\lambda}\right)^{*}=\left(f^{*}\right)_{\lambda},
$$

so we write these identical terms conveniently as $f_{\lambda}^{*}$.

We shall compute explicitly the proximal average of some indicator functions by specializing the following result. 
EXAMPLE 4.5. Let $C_{0}$ and $C_{1}$ be two nonempty closed convex subsets of $X$, and let $\lambda \in] 0,1\left[\right.$. Set $f_{0}:=\iota_{C_{0}}, f_{1}:=\iota_{C_{1}}$, and $f_{\lambda}:=\mathcal{P}\left(f_{0}, \lambda, f_{1}\right)$. Then for every $x \in X$,

$f_{\lambda}(x)=(1-\lambda) \lambda \inf \left\{\frac{1}{2}\left\|c_{0}-c_{1}\right\|^{2} \mid(1-\lambda) c_{0}+\lambda c_{1}=x\right.$ and each $c_{i}$ belongs to $\left.C_{i}\right\}$,

where inf $\varnothing=+\infty$. Moreover, dom $f_{\lambda}=(1-\lambda) C_{0}+\lambda C_{1}$.

Proof. See Appendix B or [4, Theorem 6.1].

EXAMPLE 4.6 (subspace and its orthogonal complement). Let $Y$ be a subspace of $X$ and let $\lambda \in] 0,1\left[\right.$. Set $f_{0}:=\iota_{Y}, f_{1}:=\iota_{Y \perp}$, and $f_{\lambda}:=\mathcal{P}\left(f_{0}, \lambda, f_{1}\right)$. Then

$$
\left.\left.f_{\lambda}: X \rightarrow\right]-\infty,+\infty\right]: x \mapsto \frac{1}{2(1-\lambda) \lambda}\left(\lambda^{2}\left\|P_{Y} x\right\|^{2}+(1-\lambda)^{2}\left\|P_{Y \perp} x\right\|^{2}\right)
$$

where $P_{Y}$ and $P_{Y \perp}$ denote the projectors onto $Y$ and its orthogonal complement $Y^{\perp}$, respectively. Fig. 4.1 illustrates the case $Y=\mathbb{R} \times\{0\}$.

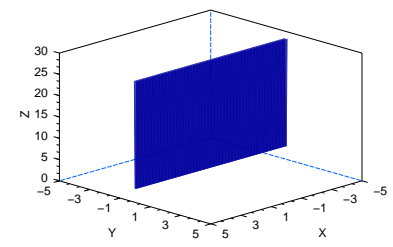

(a) $\lambda=0$

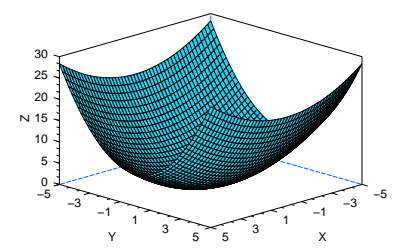

(d) $\lambda=3 / 8$

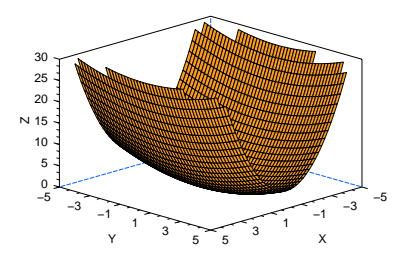

(g) $\lambda=3 / 4$

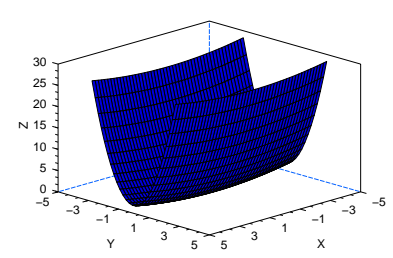

(b) $\lambda=1 / 8$

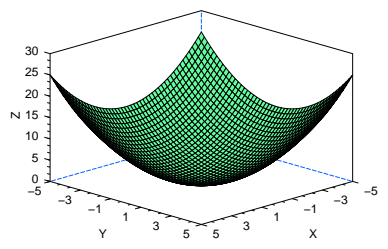

(e) $\lambda=1 / 2$

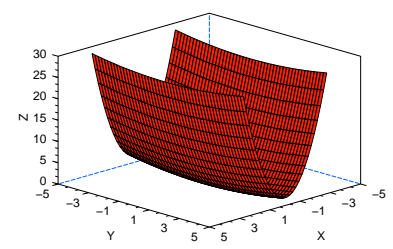

(h) $\lambda=7 / 8$

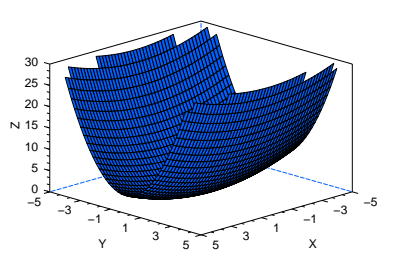

(c) $\lambda=1 / 4$

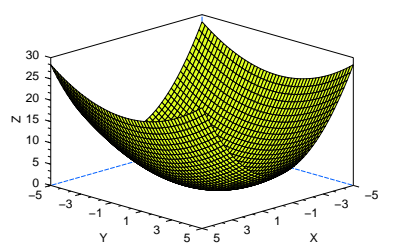

(f) $\lambda=5 / 8$

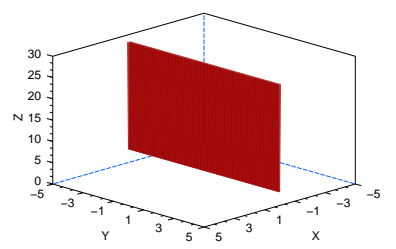

(i) $\lambda=1$

FIG. 4.1. The proximal average from the indicator of $\mathbb{R} \times\{0\}$ to the indicator of $\{0\} \times \mathbb{R}$ (see Example 4.6).

Proof. Fix $x \in X$. There is only one way to write $x$ as a sum of two vectors from $Y$ and $Y^{\perp}$, namely

$$
x=P_{Y} x+P_{Y \perp} x=(1-\lambda) P_{Y}(x /(1-\lambda))+\lambda P_{Y \perp}(x / \lambda) .
$$


In view of Example 4.5, we conclude that

$$
\begin{aligned}
f_{\lambda}(x) & =\frac{(1-\lambda) \lambda}{2}\left\|P_{Y}(x /(1-\lambda))-P_{Y \perp}(x / \lambda)\right\|^{2} . \\
& =\frac{1}{2(1-\lambda) \lambda}\left\|\lambda P_{Y} x-(1-\lambda) P_{Y} x\right\|^{2} \\
& =\frac{1}{2(1-\lambda) \lambda}\left(\lambda^{2}\left\|P_{Y} x\right\|^{2}+(1-\lambda)^{2}\left\|P_{Y \perp} x\right\|^{2}\right) .
\end{aligned}
$$

This completes the proof. $\square$

REMARK 4.7. Here are two special cases of the setting of Example 4.6.

(i) If $Y=\{0\}$, then $f_{\lambda}: x \mapsto \frac{1}{2}\left(\frac{1}{\lambda}-1\right)\|x\|^{2}$.

(ii) $f_{\frac{1}{2}}=\frac{1}{2}\|\cdot\|^{2}$.

We now verify a considerable generalization of Remark 4.7.(ii), namely that the proximal "midpoint" between a function and its Fenchel conjugate is always the energy.

Proposition 4.8. Let $f \in \mathcal{F}$. Then $\mathcal{P}\left(f, \frac{1}{2}, f^{*}\right)=\frac{1}{2}\|\cdot\|^{2}$.

Proof. Using Theorem 4.3, (3.1), and Proposition 4.2, we see that

$$
\left(\mathcal{P}\left(f, \frac{1}{2}, f^{*}\right)\right)^{*}=\mathcal{P}\left(f^{*}, \frac{1}{2}, f^{* *}\right)=\mathcal{P}\left(f^{*}, 1-\frac{1}{2}, f\right)=\mathcal{P}\left(f, \frac{1}{2}, f^{*}\right) .
$$

Thus $\mathcal{P}\left(f, \frac{1}{2}, f^{*}\right)$ is the same as its Fenchel conjugate. On the other hand, only $\frac{1}{2}\|\cdot\|^{2}$ possesses this self-conjugacy property (see (3.2)).

Another instructive case arises when we consider cones rather than subspaces. Recall that a subset $K$ of $X$ is a convex cone if $K$ is convex and $(\forall \lambda>0) \lambda K=K$. Let $K$ be a nonempty closed convex cone. Then its polar cone is

$$
K^{\ominus}:=\left\{x^{*} \in X \mid(\forall k \in K)\left\langle k, x^{*}\right\rangle \leq 0\right\} .
$$

Every subspace is a cone, but the converse is false in general (consider, e.g., $\mathbb{R}_{+}^{d}$ ). The polar cone of a subspace coincides with the orthogonal complement of the subspace; in general, these notions are different (e.g., in $\mathbb{R}$, we have $\left[0,+\infty\left[^{\ominus}=\right]-\infty\right.$, 0$]$ while $\left[0,+\infty\left[^{\perp}=\{0\}\right)\right.$. We require the notion of the projector $P_{C}$ of a nonempty closed convex set $C$ in $X$. The operator $P_{C}$ maps every $x \in X$ to its (unique) nearest point in $C: P_{C} x \in C,\left\|x-P_{C} x\right\|=\min _{c \in C}\|x-c\|$, and $\left(\forall c \in C \backslash\left\{P_{C} x\right\}\right)\left\|x-P_{C} x\right\|<\|x-c\|$.

A second glance at the proof of Example 4.6 shows that the proof was easy because the decomposition of a vector into a sum of two vectors, one taken from the subspace and the other from the orthogonal complement, is unique. Decompositions with respect to cones need not be unique: e.g., if $K=\left[0,+\infty\left[\right.\right.$ in $\mathbb{R}$, then $K^{\ominus}=-K$ yet $2=2+0=3+(-1)$ can be written in more than one way as the sum of two elements in $K$ and $K^{\ominus}$, respectively. However, it turns out that Example 4.6 holds true for cones.

EXAMPLE 4.9 (cone and polar cone). Let $K$ be a nonempty closed convex cone in $X$ and let $\lambda \in] 0,1\left[\right.$. Set $f_{0}:=\iota_{K}, f_{1}:=\iota_{K} \ominus$, and $f_{\lambda}:=\mathcal{P}\left(f_{0}, \lambda, f_{1}\right)$. Then

$$
\left.\left.f_{\lambda}: X \rightarrow\right]-\infty,+\infty\right]: x \mapsto \frac{1}{2(1-\lambda) \lambda}\left(\lambda^{2}\left\|P_{K} x\right\|^{2}+(1-\lambda)^{2}\left\|P_{K} \ominus x\right\|^{2}\right)
$$

Proof. See Appendix C.

REMARK 4.10. Consider Example 4.9. Even though the given functions $f_{0}$ and $f_{1}$ are indicator functions, the proximal average $f_{\lambda}$ is not an indicator function. 
We conclude this section with observations that are required in later sections. We start with a result that is complementary to the "Moreover" part of Example 4.5.

Theorem 4.11. Let $f_{0} \in \mathcal{F}$, let $f_{1} \in \mathcal{F}$, and let $\left.\lambda \in\right] 0,1\left[\right.$. Set $f_{\lambda}:=\mathcal{P}\left(f_{0}, \lambda, f_{1}\right)$. Then the closure and the interior of $\operatorname{dom} f_{\lambda}$ satisfy

$$
\overline{\operatorname{dom} f_{\lambda}}=\overline{(1-\lambda) \operatorname{dom} f_{0}+\lambda \operatorname{dom} f_{1}}
$$

and

$$
\operatorname{int} \operatorname{dom} f_{\lambda}=\operatorname{int}\left((1-\lambda) \operatorname{dom} f_{0}+\lambda \operatorname{dom} f_{1}\right)
$$

Proof. See Appendix D. $\square$

Corollary 4.12. Let $f_{0} \in \mathcal{F}$, let $f_{1} \in \mathcal{F}$, and let $\left.\lambda \in\right] 0,1\left[\right.$. Suppose that $f_{0}$ or $f_{1}$ has full domain. Then $\mathcal{P}\left(f_{0}, \lambda, f_{1}\right)$ has full domain as well.

Proof. Assume that $f_{0}$ has full domain. Then int $\operatorname{dom} f_{\lambda}=\operatorname{int}\left((1-\lambda) \operatorname{dom} f_{0}+\right.$ $\left.\lambda \operatorname{dom} f_{1}\right)=\operatorname{int}\left((1-\lambda) X+\lambda \operatorname{dom} f_{1}\right)=X$. $\mathrm{R}$

REMARK 4.13. Theorem 4.11 makes it clear why we observed in Fig. 1.2 that $\mathcal{P}\left(f_{0}, \lambda, f_{1}\right)$ has full domain for $\left.\lambda \in\right] 0,1[$. The results in this section admit sharpenings as well as extensions to a general (possibly infinite-dimensional) Hilbert space setting; the required results from convex analysis can be found in [23].

Proposition 4.14 (proximal average vs arithmetic average). Let $f_{0} \in \mathcal{F}$, let $f_{1} \in \mathcal{F}$, and let $\left.\lambda \in\right] 0,1\left[\right.$. Suppose that $0 \leq f_{0}, 0 \leq f_{1}$, and set $f_{\lambda}:=\mathcal{P}\left(f_{0}, \lambda, f_{1}\right)$. Then

$$
0 \leq f_{\lambda} \leq(1-\lambda) f_{0}+\lambda f_{1}
$$

Proof. Set $q:=\frac{1}{2}\|\cdot\|^{2}$. Clearly, $q \leq q+f_{0}$ and $q \leq q+f_{1}$. Conjugate these inequalities, scale the results (by $1-\lambda$ and $\lambda$, respectively), and then add to obtain $q \geq(1-\lambda)\left(q+f_{0}\right)^{*}+\lambda\left(q+f_{1}\right)^{*}$. Conjugate one more time to obtain $q \leq\left((1-\lambda)\left(q+f_{0}\right)^{*}+\lambda\left(q+f_{1}\right)^{*}\right)^{*}=f_{\lambda}+q$. It follows that

$$
\begin{aligned}
q \leq f_{\lambda}+q & =\left((1-\lambda)\left(q+f_{0}\right)^{*}+\lambda\left(q+f_{1}\right)^{*}\right)^{*} \\
& \leq(1-\lambda)\left(q+f_{0}\right)^{* *}+\lambda\left(q+f_{1}\right)^{* *} \\
& =q+(1-\lambda) f_{0}+\lambda f_{1} .
\end{aligned}
$$

The result follows by subtracting $q$.

REMARK 4.15. Suppose that $f_{\text {lower }}, f_{0}, f_{1}$, and $f_{\text {upper }}$ belong to $\mathcal{F}$ and that $f_{\text {lower }} \leq \min \left\{f_{0}, f_{1}\right\} \leq \max \left\{f_{0}, f_{1}\right\} \leq f_{\text {upper }}$. We leave it to the reader to show that

$$
(\forall \lambda \in] 0,1[) \quad f_{\text {lower }} \leq f_{\lambda} \leq f_{\text {upper }} .
$$

\section{Continuity and Homotopy.}

The examples in the preceding sections show that the proximal average is not pointwise continuous, i.e., for $f_{0} \in \mathcal{F}, f_{1} \in \mathcal{F}$, and $x \in X$, the mapping $[0,1] \rightarrow$ ]$-\infty,+\infty]: \lambda \mapsto \mathcal{P}\left(f_{0}, \lambda, f_{1}\right)(x)$ is in general not continuous. Nonetheless, the above examples and figures seem to suggest that some form of continuity is present. This is indeed the case when we use the epi-topology, a basic tool of modern variational analysis. Especially in our finite-dimensional setting, the corresponding theory is well 
developed, beautiful, and ready to use. We will draw on results from the seminal book by Rockafellar and Wets [21].

DEFINITION 5.1 (epi-convergence and epi-topology). Let $f$ and $\left(f_{n}\right)_{n \in \mathbb{N}}$ be functions from $X$ to $]-\infty,+\infty]$. Then $\left(f_{n}\right)_{n \in \mathbb{N}}$ epi-converges to $f$, in symbols, $f_{n} \stackrel{\mathrm{e}}{\rightarrow} f$, if the following hold for every $x \in X$.

(i) For every sequence $\left(x_{n}\right)_{n \in \mathbb{N}}$ in $X$ converging to $x$, one has $f(x) \leq \underline{\lim } f_{n}\left(x_{n}\right)$.

(ii) There exists a sequence $\left(y_{n}\right)_{n \in \mathbb{N}}$ in $X$ converging to $x$ such that $\overline{\lim }_{n}\left(y_{n}\right) \leq$ $f(x)$.

The epi-topology is the topology induced by epi-convergence.

The notion of epi-convergence is especially useful in optimization. It has its roots in the theory of convergence of epigraphs (see (2.3)), viewed as subsets of $X \times \mathbb{R}$; the reader is referred to [21, Chapter 7] for further information. The following example (see [21, Section 7.B]) illustrates that epi-convergence is independent of the classical notion of pointwise convergence.

EXAmple 5.2. For every $n \in \mathbb{N}$, define

$$
f_{n}: \mathbb{R} \rightarrow \mathbb{R}: x \mapsto \begin{cases}0, & \text { if } x=1 /(n+1) ; \\ 1, & \text { otherwise. }\end{cases}
$$

Then the pointwise-limit of $\left(f_{n}\right)_{n \in \mathbb{N}}$ is the function that is identically equal to 1; however, the epi-limit of $\left(f_{n}\right)_{n \in \mathbb{N}}$ is the function

$$
f: \mathbb{R} \rightarrow \mathbb{R}: x \mapsto \begin{cases}0, & \text { if } x=0 ; \\ 1, & \text { otherwise } .\end{cases}
$$

Proof. The statement concerning the pointwise-limit is clear. Now fix $x \in \mathbb{R}$. Let $\left(x_{n}\right)_{n \in \mathbb{N}}$ be a sequence in $\mathbb{R}$ converging to $x$.

Case 1. $x=0$. Then $f(x)=f(0)=0 \leq \underline{\lim } f_{n}\left(x_{n}\right)$. Set $(\forall n \in \mathbb{N}) y_{n}:=1 /(n+1)$. Then $y_{n} \rightarrow x$ and $(\forall n \in \mathbb{N}) f_{n}\left(y_{n}\right)=0$. Hence $\overline{\lim } f_{n}\left(y_{n}\right)=\varlimsup \lim 0=0=f(0)=f(x)$. This verifies items (i) and (ii) of Definition 5.1 at $x=0$.

Case 2. $x \neq 0$. Then eventually $\left|x_{n}\right| \geq|x| / 2>0$ and, in turn, eventually $f_{n}\left(x_{n}\right)=1$. It follows that $f(x)=1=\lim f_{n}\left(x_{n}\right)=\underline{\lim } f_{n}\left(x_{n}\right)$. Set $(\forall n \in \mathbb{N})$ $y_{n}:=x$. Then $y_{n} \rightarrow x$ and eventually $f_{n}\left(y_{n}\right)=f_{n}(x)=1$. Thus $\varlimsup$ im $f_{n}\left(y_{n}\right)=\varlimsup \lim 1=$ $1=f(x)$. This verifies items (i) and (ii) of Definition 5.1 at $x$.

Hence in either case, items (i) and (ii) of Definition 5.1 hold, i.e., $f_{n} \stackrel{\text { e }}{\rightarrow} f$.

The following fact implies the continuity — with respect to the epi-topology of various operations.

FACT 5.3. Let $\left(f_{n}\right)_{n \in \mathbb{N}}$ be a sequence in $\mathcal{F}$ such that $\left(f_{n}\right)_{n \in \mathbb{N}}$ epi-converges to a function $f \in \mathcal{F}$, set $q:=\frac{1}{2}\|\cdot\|^{2}$, let $\left(\mu_{n}\right)_{n \in \mathbb{N}}$ be a sequence in $[0,1]$ converging to $\mu$, and let $\left(g_{n}\right)_{n \in \mathbb{N}}$ be a sequence in $\mathcal{F}$ such that $\left(g_{n}\right)_{n \in \mathbb{N}}$ epi-converges to a function $g \in \mathcal{F}$. Then the following hold.

(i) $f_{n} \pm q \stackrel{\mathrm{e}}{\rightarrow} f \pm q$.

(ii) $f_{n}^{*} \stackrel{\mathrm{e}}{\rightarrow} f^{*}$.

(iii) $\mu_{n} f_{n} \stackrel{\mathrm{e}}{\rightarrow} \mu f$ provided that $\mu>0$.

(iv) $\mu_{n} f_{n} \stackrel{\mathrm{e}}{\rightarrow} \mu f$ provided that $\mu=0$ and $\operatorname{dom} f=X$.

(v) $f_{n}+g_{n} \stackrel{\mathrm{e}}{\rightarrow} f+g$ provided that $0 \in \operatorname{int}(\operatorname{dom} f-\operatorname{dom} g)$.

Proof. (i): This is a consequence of [21, Exercise 7.8(a)].

(ii): See [21, Theorem 11.34].

(iii): See [21, Exercise 7.8(d)]. 
(iv): Let $\left(x_{n}\right)_{n \in \mathbb{N}}$ be a sequence in $X$ converging to $x$. Then $-\infty<f(x) \leq$ $\underline{\lim } f_{n}\left(x_{n}\right)$ and hence $(0 \cdot f)(x)=0 \leq \underline{\lim }\left(\mu_{n} f_{n}\right)\left(x_{n}\right)$. On the other hand, there exists a sequence $\left(y_{n}\right)_{n \in \mathbb{N}}$ in $X$ such that $y_{n} \rightarrow y$ and $\varlimsup_{\lim } f_{n}\left(y_{n}\right) \leq f(x)<+\infty$. Hence $\varlimsup\left(\mu_{n} f_{n}\right)\left(y_{n}\right) \leq 0=(0 \cdot f)(x)$. Altogether, $\left(\mu_{n} f_{n}\right)_{n \in \mathbb{N}}$ epi-converges to $0 \cdot f$.

(v): See [21, Exercise 7.47(b)].

Based on Fact 5.3, we are now able to establish the continuity of the proximal average operator.

THEOREM 5.4 (continuity). Suppose that $\mathcal{F}$ is equipped with the epi-topology. Then the proximal average operator $\mathcal{P}: \mathcal{F} \times[0,1] \times \mathcal{F} \rightarrow \mathcal{F}$ is continuous.

Proof. Let $\left(f_{n}\right)_{n \in \mathbb{N}}$ and $\left(g_{n}\right)_{n \in \mathbb{N}}$ be sequences in $\mathcal{F}$, let $f$ and $g$ be in $\mathcal{F}$, let $\left(\lambda_{n}\right)_{n \in \mathbb{N}}$ be a sequence in $[0,1]$, and let $\lambda \in[0,1]$. Assume that

$$
f_{n} \stackrel{\mathrm{e}}{\rightarrow} f, \quad g_{n} \stackrel{\mathrm{e}}{\rightarrow} g, \quad \text { and } \quad \lambda_{n} \rightarrow \lambda .
$$

Set $q:=\frac{1}{2}\|\cdot\|^{2}$. Fact 5.3.(i) implies that $f_{n}+q \stackrel{\mathrm{e}}{\rightarrow} f+q$ and $g_{n}+q \stackrel{\mathrm{e}}{\rightarrow} g+q$. Now Fact 5.3.(ii) yields $\left(f_{n}+q\right)^{*} \stackrel{\mathrm{e}}{\rightarrow}(f+q)^{*}$ and $\left(g_{n}+q\right)^{*} \stackrel{\mathrm{e}}{\rightarrow}(g+q)^{*}$. Since $\operatorname{dom}(f+g)^{*}=X$ and $\operatorname{dom}(g+q)^{*}=X$, Fact 5.3.(iii)\&(iv) show that $\left(1-\lambda_{n}\right)\left(f_{n}+q\right)^{*} \stackrel{\mathrm{e}}{\rightarrow}(1-\lambda)(f+q)^{*}$ and $\lambda_{n}\left(g_{n}+q\right)^{*} \stackrel{\mathrm{e}}{\rightarrow} \lambda(g+q)^{*}$. Because $(1-\lambda)(f+q)^{*}$ and $\lambda(g+q)^{*}$ each has full domain, we deduce from Fact 5.3.(v) that $\left(1-\lambda_{n}\right)\left(f_{n}+q\right)^{*}+\lambda_{n}\left(g_{n}+q\right)^{*} \stackrel{\mathrm{e}}{\rightarrow}(1-\lambda)(f+q)^{*}+\lambda(g+$ $q)^{*}$. By Fact 5.3.(ii), $\left(\left(1-\lambda_{n}\right)\left(f_{n}+q\right)^{*}+\lambda_{n}\left(g_{n}+q\right)^{*}\right)^{*} \stackrel{\mathrm{e}}{\rightarrow}\left((1-\lambda)(f+q)^{*}+\lambda(g+q)^{*}\right)^{*}$. Finally, Fact 5.3.(i) implies that

$$
\left(\left(1-\lambda_{n}\right)\left(f_{n}+q\right)^{*}+\lambda_{n}\left(g_{n}+q\right)^{*}\right)^{*}-q \stackrel{\mathrm{e}}{\rightarrow}\left((1-\lambda)(f+q)^{*}+\lambda(g+q)^{*}\right)^{*}-q,
$$

i.e., $\mathcal{P}\left(f_{n}, \lambda_{n}, g_{n}\right) \stackrel{\text { e }}{\rightarrow} \mathcal{P}(f, \lambda, g)$.

Corollary 5.5 (homotopy). Let $f_{0} \in \mathcal{F}$ and let $f_{1} \in \mathcal{F}$. Then

$$
\mathcal{P}\left(f_{0}, \cdot, f_{1}\right):[0,1] \rightarrow \mathcal{F}
$$

provides a homotopy between $f_{0}$ and $f_{1}$. Consequently, all functions in $\mathcal{F}$ are homotopic.

REMARK 5.6. In infinite-dimensional spaces, the theory is more complicated and there are a number of topologies one might consider (most of which coincide in finite dimensions). We believe the most useful topology for carrying the results of this section over is the Attouch-Wets topology, for which addition and Fenchel conjugation are continuous operations. We refer the interested reader to the books $[1,6,15]$ as well as to the articles $[2,19]$.

\section{Inheritance.}

Recall that $f \in \mathcal{F}$ is strictly convex, if

$$
\left.\begin{array}{c}
x \in \operatorname{dom} f \\
y \in \operatorname{dom} f \\
\lambda \in] 0,1[ \\
x \neq y
\end{array}\right\} \Rightarrow f((1-\lambda) x+\lambda y)<(1-\lambda) f(x)+\lambda f(y) .
$$

Strict convexity is important in optimization because it guarantees that there is at most one minimizer of any objective function with this property. Fenchel conjugacy shows that strict convexity and differentiability are dual to each other in the following immensely useful and beautiful sense. 
FACT 6.1. Let $f \in \mathcal{F}$ such that both $f$ and $f^{*}$ have full domain. Then

$f$ is differentiable $\Leftrightarrow f^{*}$ is strictly convex.

We now show that the proximal average inherits good properties such as differentiability or strict convexity from the given functions.

Theorem 6.2 (inheritance). Let $f_{0} \in \mathcal{F}$, let $f_{1} \in \mathcal{F}$, and let $\left.\lambda \in\right] 0,1[$. Set $f_{\lambda}:=\mathcal{P}\left(f_{0}, \lambda, f_{1}\right)$. Suppose that $f_{0}$ or $f_{1}$ has full domain, and that $f_{0}^{*}$ or $f_{1}^{*}$ has full domain. Then the following hold.

(i) Both $f_{\lambda}$ and $f_{\lambda}^{*}$ have full domain.

(ii) If $f_{0}$ or $f_{1}$ is differentiable everywhere, then so is $f_{\lambda}$.

(iii) If $f_{0}$ or $f_{1}$ is strictly convex and its Fenchel conjugate has full domain, then $f_{\lambda}$ is strictly convex.

Proof. (i): $f_{\lambda}$ has full domain by Corollary 4.12. A dual argument (recall Remark 4.4) shows that $f_{\lambda}^{*}$ has full domain as well.

(ii): Assume that $f_{0}$ is differentiable with full domain. Then so is $f_{0}+q$ and hence (by Fact 6.1) $\left(f_{0}+q\right)^{*}=f_{0}^{*} \square q$ is strictly convex with full domain. Thus $(1-\lambda)\left(f_{0}+q\right)^{*}+\lambda\left(f_{1}+q\right)^{*}$ is strictly convex with full domain. Item (i) and Fact 6.1 imply that $f_{\lambda}+q$ is differentiable with full domain. Therefore, $f_{\lambda}$ is differentiable with full domain.

(iii): Assume that $f_{0}$ is strictly convex and that $\operatorname{dom} f_{0}^{*}=X$. Then $f_{0}^{*}$ is differentiable everywhere and hence so is $f_{\lambda}^{*}$ (by (ii) applied to $f_{0}^{*}$ and $f_{1}^{*}$ ). We conclude that $f_{\lambda}$ is strictly convex.

Corollary 6.3. Let $f_{0} \in \mathcal{F}$, let $f_{1} \in \mathcal{F}$, and let $\left.\lambda \in\right] 0,1\left[\right.$. Suppose that $f_{0}, f_{1}$, and their Fenchel conjugates have full domain, that $f_{0}$ is strictly convex, and that $f_{1}$ is differentiable. Set $f_{\lambda}:=\mathcal{P}\left(f_{0}, \lambda, f_{1}\right)$. Then both $f_{\lambda}$ and $f_{\lambda}^{*}$ are differentiable and strictly convex.

REMARK 6.4. Assume that the hypothesis of Corollary 6.3 holds and that $f_{0}$ is not differentiable. The conclusion of Corollary 6.3 guarantees that the proximal average $f_{\lambda}$ is differentiable. In contrast, the arithmetic average $(1-\lambda) f_{0}+\lambda f_{1}$ is not differentiable. This illustrates another advantage of the proximal average over the arithmetic average. Note that both averages produce a strictly convex function.

Recall that a norm $\llbracket \cdot]($ on $X)$ is a function $X \rightarrow \mathbb{R}$ satisfying the following four properties: $(\forall x \in X) \llbracket x \rrbracket \geq 0 ;(\forall x \in X) \rrbracket x \rrbracket=0 \Leftrightarrow x=0 ;(\forall x \in X)(\forall r \in \mathbb{R})$ $\llbracket r x \rrbracket=|r| \rrbracket x \rrbracket$; and $(\forall x \in X)(\forall y \in X) \llbracket x+y \rrbracket \leq \rrbracket x \rrbracket+\llbracket y \rrbracket$. Every norm $\rrbracket \cdot \rrbracket$ can be written as $\square \cdot \square=\sqrt{2 f}$, where the unique function $f \in \mathcal{F}$ is nonnegative, it vanishes only at 0 , and it is quadratic-homogeneous, i.e., $(\forall x \in X)(\forall r \in \mathbb{R}) f(r x)=r^{2} f(x)$; we shall say that $f$ is the function associated with $\llbracket \cdot \square$, and that $\square \cdot \square$ is the norm associated with $f$. Let [ . [ be a norm with associated function $f$. Then $f^{*}$ is associated with the corresponding dual norm $\rrbracket \cdot \rrbracket^{\circ}: X \rightarrow \mathbb{R}: x^{*} \mapsto \sup \left\{\left\langle x, x^{*}\right\rangle \mid \rrbracket x \rrbracket \leq 1\right\}$. (See $[20$,

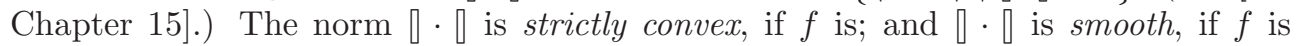
differentiable everywhere. Note that Fact 6.1 implies that [ . [ is strictly convex $\Leftrightarrow$ $\square \cdot \square^{\circ}$ is smooth, and that $\square \cdot \square$ is smooth $\Leftrightarrow \square \cdot \square^{\circ}$ is strictly convex.

We now show that the proximal average can be employed to create a new norm that inherits good properties from two given norms.

Theorem 6.5 (renorming). Let $\square \cdot \rrbracket_{0}$ and $\square \cdot \square_{1}$ be two norms on $X$, and denote their associated functions by $f_{0}$ and $f_{1}$. Let $\left.\lambda \in\right] 0,1\left[\right.$ and set $f_{\lambda}:=\mathcal{P}\left(f_{0}, \lambda, f_{1}\right)$. Then $f_{\lambda}$ is associated with some norm $\square \cdot \square_{\lambda}, f_{\lambda}^{*}$ is associated with $\square \cdot \square_{\lambda}^{\circ}$, and

$$
0 \leq\left(\frac{1}{1-\lambda} f_{0}\right) \square\left(\frac{1}{\lambda} f_{1}\right) \leq f_{\lambda} \leq(1-\lambda) f_{0}+\lambda f_{1} .
$$


If $\square \cdot \square_{0}$ is strictly convex and $\square \cdot \square_{1}$ is smooth, then $\square \cdot \square_{\lambda}$ and $\square \cdot \square_{\lambda}^{\circ}$ are strictly convex and smooth.

Proof. Since $0 \leq f_{0}$ and $0 \leq f_{1}$, the first inequality in (6.1) follows immediately. The third inequality in (6.1) was already recorded in Proposition 4.14. Applying this proposition to $f_{0}^{*}$ and $f_{1}^{*}$, which are the functions associated with the dual norms $\llbracket \cdot \rrbracket_{0}^{\circ}$ and $\llbracket \cdot \rrbracket_{1}^{\circ}$, we see that $f_{\lambda}^{*} \leq(1-\lambda) f_{0}^{*}+\lambda f_{1}^{*}$. Conjugate this inequality to obtain

$$
f_{\lambda} \geq\left(\left((1-\lambda) f_{0}^{*}\right)^{*}\right) \square\left(\left(\lambda f_{1}^{*}\right)^{*}\right)=\left((1-\lambda) f_{0}(\cdot /(1-\lambda))\right) \square\left(\lambda f_{0}(\cdot / \lambda)\right) .
$$

The second inequality in (6.1) thus follows from the quadratic homogeneity of $f_{0}$ and $f_{1}$. Let us show that $f_{\lambda}$ is associated with some norm $\llbracket \cdot \rrbracket_{\lambda}$. It is clear that $f_{\lambda}$ belongs to $\mathcal{F}$, and (6.1) yields $0 \leq f_{\lambda}$. Suppose that $x \in X$ satisfies $f_{\lambda}(x)=0$. Since $f_{0}$ and $f_{1}$ are both coercive (i.e., each $\lim _{\|x\| \rightarrow+\infty} f_{i}(x)=+\infty$ ), the infimal convolution of (6.1) is exact at $x$ (i.e., the infimum in the definition of the infimal convolution at the point $x$ is actually attained, i.e., the infimum is a minimum). Hence there exist vectors $x_{0}$ and $x_{1}$ in $X$ such that $x=x_{0}+x_{1}$ and $0 \leq(1 /(1-\lambda)) f_{0}\left(x_{0}\right)+(1 / \lambda) f_{1}\left(x_{1}\right) \leq f_{\lambda}(x)=0$; Thus $f_{0}\left(x_{0}\right)=f_{1}\left(x_{1}\right)=0$, which implies $x_{0}=0=x_{1}$ and further $x=0$. It follows that $f_{\lambda}$ vanishes only at 0 . The definition of $f_{\lambda}$ now leads readily to the quadratic homogeneity of $f_{\lambda}$. Therefore, $f_{\lambda}$ is associated with some norm $\square \cdot \eta_{\lambda}$ and $f_{\lambda}^{*}$ is associated with $\square \cdot \square_{\lambda}^{\circ}$. Finally, we assume that $f_{0}$ is strictly convex and $f_{1}$ is differentiable everywhere. In view of Corollary $6.3, f_{\lambda}$ and $f_{\lambda}^{*}$ are strictly convex and differentiable; equivalently, [ $\cdot \rrbracket_{\lambda}$ and $] \cdot \square_{\lambda}^{\circ}$ are strictly convex and smooth.

Define two norms at any point $\left(x_{1}, x_{2}\right) \in \mathbb{R}^{2}$ via

$$
\llbracket\left(x_{1}, x_{2}\right) \rrbracket_{0}= \begin{cases}\frac{1}{2}\left|x_{1}\right|, & \text { if }\left|x_{1}\right|>2\left|x_{2}\right| ; \\ \frac{1}{2}\left|x_{2}\right|, & \text { if }\left|x_{2}\right|>2\left|x_{1}\right| ; \\ \left|x_{1}\right|+\left|x_{2}\right|-\sqrt{2\left|x_{1}\right|\left|x_{2}\right|}, & \text { otherwise, }\end{cases}
$$

and

$$
\llbracket\left(x_{1}, x_{2}\right) \rrbracket_{1}=\frac{\left|x_{1}\right|+\sqrt{4\left|x_{1}\right|^{2}+3\left|x_{2}\right|^{2}}}{3} .
$$

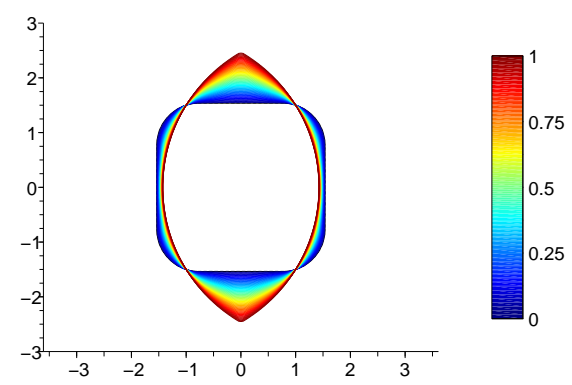

FIG. 6.1. An illustration of Theorem 6.5 when $\square \cdot \square_{0}$ and $\square \cdot \square_{1}$ are defined by (6.2) and (6.3), respectively. The unit spheres of the norms $\left[\cdot \square_{\lambda}\right.$, where $\lambda \in[0,1]$, are visualized.

Note that $\square \cdot \square_{0}$ is smooth but not strictly convex, and that $\square \cdot \square_{1}$ is strictly convex but not smooth. The unit spheres of these norms - as well as those of all norms $\llbracket \cdot \square_{\lambda}$ obtained by Theorem 6.5 - are illustrated in Fig. 6.1. As predicted by the theory, the intermediate norms $\llbracket \cdot \rrbracket_{\lambda}$ are both smooth and strictly convex. 


\section{Exercises.}

In this section, we present a collection of examples some of which were previously considered by the first-named author and A. Jarvis [14]. We do leave the details of these examples to the interested reader as exercises and we encourage him/her to utilize available software such as $[5,7,12,16,17]$, which was very beneficial for the creation of the examples presented in this paper.

In all these examples, we shall specify two functions $f_{0}$ and $f_{1}$ in $\mathcal{F}$, and we shall assume that

$$
\lambda \in] 0,1\left[\text { and } f_{\lambda}:=\mathcal{P}\left(f_{0}, \lambda, f_{1}\right) .\right.
$$

The first example shows that the proximal average coincides with the arithmetic average if one function is simply a (vertical) translate of the other.

EXAmple 7.1. Let $f_{0} \in \mathcal{F}$, let $\gamma \in \mathbb{R}$, and set $f_{1}:=f_{0}+\gamma$. Then $f_{\lambda}=f_{0}+\lambda \gamma=$ $(1-\lambda) f_{0}+\lambda f_{1}$.

On the other hand, if we consider the proximal average between 0 and a nonzero linear functional, then we will not obtain the arithmetic average.

ExAMPle 7.2. Let $a \in X \backslash\{0\}$, set $f_{0}:=0$ and $f_{1}:=\langle\cdot, a\rangle$. Then $f_{\lambda}=$ $\lambda\langle\cdot, a\rangle-(1-\lambda) \lambda \frac{1}{2}\|a\|^{2}$.

Next let us demonstrate that simple scaling may lead to relatively complicated proximal averages.

EXAmPle 7.3. Let $\alpha>0$, set $f_{0}:=\frac{1}{2}\|\cdot\|^{2}$ and $f_{1}:=\alpha \frac{1}{2}\|\cdot\|^{2}$. Then

$$
f_{\lambda}=\frac{(1+\lambda) \alpha+1-\lambda}{(1-\lambda) \alpha+1+\lambda} \frac{1}{2}\|\cdot\|^{2} .
$$

By discussing the case of true quadratics and linear functions separately, it is possible to obtain the following explicit formula for transforming between two quadratics. Note that the proximal average is a quadratic as well unless both functions are linear.

Example 7.4 (two quadratics). Let $\alpha_{0} \geq 0$, let $\alpha_{1} \geq 0$, let $b_{0} \in X$, let $b_{1} \in X$, let $\gamma_{0} \in \mathbb{R}$, and let $\gamma_{1} \in \mathbb{R}$. Define $f_{0}: x \mapsto \alpha_{0}\|x\|^{2}+\left\langle x, b_{0}\right\rangle+\gamma_{0}$ and $f_{1}: x \mapsto$ $\alpha_{1}\|x\|^{2}+\left\langle x, b_{1}\right\rangle+\gamma_{1}$, and set

(i) $\delta_{\lambda}:=1+2\left(\lambda \alpha_{0}+(1-\lambda) \alpha_{1}\right)$,

(ii) $\alpha_{\lambda}:=\frac{2 \alpha_{0} \alpha_{1}+(1-\lambda) \alpha_{0}+\lambda \alpha_{1}}{\delta_{\lambda}}$,

(iii) $b_{\lambda}:=\frac{(1-\lambda)\left(2 \alpha_{1}+1\right) b_{0}+\lambda\left(2 \alpha_{0}+1\right) b_{1}}{\delta_{\lambda}}$, and Then

(iv) $\gamma_{\lambda}:=(1-\lambda) \gamma_{0}+\lambda \gamma_{1}-\frac{(1-\lambda) \lambda\left\|b_{0}-b_{1}\right\|^{2}}{2 \delta_{\lambda}}$.

$$
f_{\lambda}: x \mapsto \alpha_{\lambda}\|x\|^{2}+\left\langle x, b_{\lambda}\right\rangle+\gamma_{\lambda} .
$$

Fig. 1.1(b) illustrates the case $\alpha_{0}=0, b_{0}=1, \gamma_{0}=2$, and $\alpha_{1}=1, b_{1}=0, \gamma_{1}=0$.

The transformation from a quadratic to a singleton indicator follows along a quadratic path.

ExAmple 7.5. Let $\alpha_{0} \geq 0$, let $b_{0} \in X$, let $b_{1} \in X$, let $\gamma_{0} \in \mathbb{R}$, and let $\gamma_{1} \in \mathbb{R}$. Define $f_{0}: x \mapsto \alpha_{0}\|x\|^{2}+\left\langle x, b_{0}\right\rangle+\gamma_{0}$ and $f_{1}:=\iota_{\left\{b_{1}\right\}}+\gamma_{1}$, and set

(i) $\alpha_{\lambda}:=\frac{2 \alpha_{0}+\lambda}{2(1-\lambda)}$, 
Then

(ii) $b_{\lambda}:=\frac{(1-\lambda) b_{0}-\lambda\left(1+2 \alpha_{0}\right) b_{1}}{1-\lambda}$, and

(iii) $\gamma_{\lambda}:=\frac{2(1-\lambda)^{2} \gamma_{0}+2 \lambda(1-\lambda) \gamma_{1}+\lambda\left\langle b_{1},\left(1+2 \lambda \alpha_{0}\right) b_{1}-2(1-\lambda) b_{0}\right\rangle}{2(1-\lambda)}$.

$$
f_{\lambda}: x \mapsto \alpha_{\lambda}\|x\|^{2}+\left\langle x, b_{\lambda}\right\rangle+\gamma_{\lambda} .
$$

Corollary 6.3 implies that for each $\lambda \in] 0,1\left[\right.$, the proximal average $f_{\lambda}$ is both strictly convex and differentiable. Fig. 3.1(b) illustrates the case when $\alpha_{0}=\frac{1}{2}, b_{0}=0, \gamma_{0}=0$, and $b_{1}=0, \gamma_{1}=1$. If $\alpha_{0}=0$, then $f_{0}$ is differentiable but not strictly convex, and $f_{1}$ is strictly convex but not differentiable.

We conclude this section with two singleton indicator functions.

ExAmple 7.6. Let $b_{0} \in X$, let $b_{1} \in X$, let $\gamma_{0} \in \mathbb{R}$, and let $\gamma_{1} \in \mathbb{R}$. Set $f_{0}:=\iota_{\left\{b_{0}\right\}}+\gamma_{0}$ and $f_{1}:=\iota_{\left\{b_{1}\right\}}+\gamma_{1}$. Then

$$
f_{\lambda}=\iota_{\left\{(1-\lambda) b_{0}+\lambda b_{1}\right\}}+(1-\lambda) \gamma_{0}+\lambda \gamma_{1}+\frac{(1-\lambda) \lambda}{2}\left\|b_{0}-b_{1}\right\|^{2} .
$$

\section{Appendix A. Proof of Theorem 4.3.}

The result is clear if $\lambda \in\{0,1\}$ so we assume that $\lambda \in] 0,1\left[\right.$. Set $\lambda_{0}:=(1-\lambda)$ and $\lambda_{1}:=\lambda$, and set $q:=\frac{1}{2}\|\cdot\|^{2}$ so that $q=q^{*}($ see $(3.2))$ and $\operatorname{dom} q=X$. For $i \in\{0,1\}$, set $g_{i}:=\lambda_{i}\left(f_{i}+q\right)^{*}=\lambda_{i}\left(f_{i}^{*} \square q\right)$. Now each $f_{i}^{*}$ belongs to $\mathcal{F}$, hence so does each $g_{i}$ and each $g_{i}$ has full domain. Thus $g_{0}+g_{1} \in \mathcal{F}$ and $\operatorname{dom}\left(g_{0}+g_{1}\right)=X$. It follows that $\left(g_{0}+g_{1}\right)^{*}$ is proper. Now fix $x^{*} \in X^{*}$. Then convex calculus yields

$$
\begin{aligned}
\left(\mathcal{P}\left(f_{0}, \lambda, f_{1}\right)\right)^{*}\left(x^{*}\right) & =\left(\left(g_{0}+g_{1}\right)^{*}-q\right)^{*}\left(x^{*}\right) \\
& =\sup _{z^{*}}\left(g_{0}+g_{1}\right)^{* *}\left(x^{*}+z^{*}\right)-q\left(z^{*}\right) \\
& =\sup _{z^{*}}\left(g_{0}+g_{1}\right)\left(x^{*}+z^{*}\right)-q\left(z^{*}\right) \\
& =\sup _{y^{*}}\left(g_{0}+g_{1}\right)\left(y^{*}\right)-q\left(y^{*}-x^{*}\right) \\
& =\sup _{y^{*}} \lambda_{0}\left(f_{0}^{*} \square q\right)\left(y^{*}\right)+\lambda_{1}\left(f_{1}^{*} \square q\right)\left(y^{*}\right)-\left(q\left(y^{*}\right)+q\left(x^{*}\right)-\left\langle x^{*}, y^{*}\right\rangle\right) \\
& =\sup _{y^{*}}\left(\lambda_{0}\left(q\left(y^{*}\right)-\left(f_{0} \square q\right)\left(y^{*}\right)\right)+\lambda_{1}\left(q\left(y^{*}\right)-\left(f_{1} \square q\right)\left(y^{*}\right)\right)\right. \\
& \left.-\left(q\left(y^{*}\right)+q\left(x^{*}\right)-\left\langle y^{*}, x^{*}\right\rangle\right)\right) \\
= & -q\left(x^{*}\right)+\sup _{y^{*}}\left\langle x^{*}, y^{*}\right\rangle-\left(\lambda_{0}\left(f_{0} \square q\right)+\lambda_{1}\left(f_{0} \square q\right)\right)\left(y^{*}\right) \\
= & \left(\lambda_{0}\left(f_{0} \square q\right)+\lambda_{1}\left(f_{1} \square q\right)\right)^{*}\left(x^{*}\right)-q\left(x^{*}\right) \\
= & \left(\lambda_{0}\left(f_{0}^{*}+q\right)^{*}+\lambda_{1}\left(f_{1}^{*}+q\right)^{*}\right)^{*}\left(x^{*}\right)-q\left(x^{*}\right) \\
= & \left((1-\lambda)\left(f_{0}^{*}+q\right)^{*}+\lambda\left(f_{1}^{*}+q\right)^{*}\right)^{*}\left(x^{*}\right)-q\left(x^{*}\right) \\
= & \left(\mathcal{P}\left(f_{0}^{*}, \lambda, f_{1}^{*}\right)\right)\left(x^{*}\right) .
\end{aligned}
$$

This verifies (4.2). Hence

$$
\left(\mathcal{P}\left(f_{0}, \lambda, f_{1}\right)\right)^{* *}=\left(\mathcal{P}\left(f_{0}^{*}, \lambda, f_{1}^{*}\right)\right)^{*}=\mathcal{P}\left(f_{0}^{* *}, \lambda, f_{1}^{* *}\right)=\mathcal{P}\left(f_{0}, \lambda, f_{1}\right)
$$

and therefore $\mathcal{P}\left(f_{0}, \lambda, f_{1}\right) \in \mathcal{F}$. The proof of Theorem 4.3 is complete. 


\section{Appendix B. Proof of Example 4.5.}

Fix $x \in X$. Using the notation of Appendix A, we obtain

$$
\begin{aligned}
f(x) & =\left(g_{0}+g_{1}\right)^{*}(x)-q(x) \\
& =\left(g_{0}^{*} \square g_{1}^{*}\right)(x)-q(x) \\
& =\inf _{x_{0}+x_{1}=x} g_{0}^{*}\left(x_{0}\right)+g_{1}^{*}\left(x_{1}\right)-q(x) \\
& =\inf _{x_{0}+x_{1}=x} \lambda_{0}\left(\iota_{C_{0}}+q\right)\left(\lambda_{0}^{-1} x_{0}\right)+\lambda_{1}\left(\iota_{C_{1}}+q\right)\left(\lambda_{1}^{-1} x_{1}\right)-q(x) \\
& =\inf _{x_{0}+x_{1}=x \text { and each } \lambda_{i}^{-1} x_{i} \in C_{i}} \lambda_{0} q\left(\lambda_{0}^{-1} x_{0}\right)+\lambda_{1} q\left(\lambda_{1}^{-1} x_{1}\right)-q\left(x_{0}+x_{1}\right) \\
& =\inf _{\lambda_{0} c_{0}+\lambda_{1} c_{1}=x \text { and each } c_{i} \in C_{i}} \lambda_{0} q\left(c_{0}\right)+\lambda_{1} q\left(c_{1}\right)-q\left(\lambda_{0} c_{0}+\lambda_{1} c_{1}\right) \\
& ={ }_{\lambda_{0} c_{0}+\lambda_{1} c_{1}=x \text { and each } c_{i} \in C_{i}} \lambda_{0} \frac{1}{2}\left\|c_{0}\right\|^{2}+\lambda_{1} \frac{1}{2}\left\|c_{1}\right\|^{2}-\frac{1}{2}\left\|\lambda_{0} c_{0}+\lambda_{1} c_{1}\right\|^{2} \\
& =\inf _{\lambda_{0} c_{0}+\lambda_{1} c_{1}=x \text { and each } c_{i} \in C_{i}} \frac{1}{2} \lambda_{0} \lambda_{1}\left\|c_{0}-c_{1}\right\|^{2} \\
& =\inf _{(1-\lambda) c_{0}+\lambda c_{1}=x \text { and each } c_{i} \in C_{i}} \frac{1}{2}(1-\lambda) \lambda\left\|c_{0}-c_{1}\right\|^{2} .
\end{aligned}
$$

This verifies the formula for $f_{\lambda}$ and the "Moreover" part is now clear. This concludes the proof of Example 4.5.

\section{Appendix C. Proof of Example 4.9.}

We begin by stating a classical result due to Moreau. This result can be interpreted as the Pythagorean decomposition for cones.

FACT C.1. Let $K$ be a nonempty closed convex cone in $X$. Then for every $x$ in $X, x=P_{K} x+P_{K \ominus} x$ and $\left\langle P_{K} x, P_{K \ominus} x\right\rangle=0$.

We require the following consequence of Moreau's result.

Proposition C.2. Let $K$ be a nonempty closed convex cone in $X$ and let $x \in X$. Then the unique solution to the optimization problem

$$
\text { minimize }\|k\| \text { subject to } k \in K \text { and } x-k \in K^{\ominus}
$$

is the vector $P_{K} x$.

Proof. Set $K^{\oplus}=-K^{\ominus}$. By Fact C.1, $K \cap\left(x-K^{\ominus}\right)$ is nonempty and thus the optimization problem (C.1) is feasible, i.e., the minimum is not taken over the empty set. Hence (C.1) is equivalent to another feasible optimization problem, namely

$$
\text { minimize }\|k\| \text { subject to } k \in K \text { and } k \in x+K^{\oplus} \text {. }
$$

Since $K \cap\left(x+K^{\oplus}\right) \neq \varnothing$, the unique solution of (C.2) is $P_{K \cap\left(x+K^{\oplus}\right)} 0$. On the other hand, we have

$$
P_{x+K^{\oplus}} 0=x+P_{K^{\oplus}}(0-x)=x+P_{-K^{\ominus}}(-x)=x-P_{K^{\ominus}} x=P_{K} x .
$$

(See also [3, Remark 3.3].) Altogether, we conclude that $P_{K \cap\left(x+K^{\oplus}\right)} 0=P_{K} x$.

Let us now verify Example 4.9. Fix $x \in X$. Utilizing Example 4.5, Fact C.1, and 
Proposition C.2, we estimate that

$$
\begin{aligned}
f_{\lambda}(x) & \leq \frac{(1-\lambda) \lambda}{2}\left\|P_{K}\left(\frac{1}{1-\lambda} x\right)-P_{K^{\ominus}}\left(\frac{1}{\lambda} x\right)\right\|^{2} \\
& =\frac{(1-\lambda) \lambda}{2}\left(\frac{1}{(1-\lambda)^{2}}\left\|P_{K} x\right\|^{2}+\frac{1}{\lambda^{2}}\left\|P_{K \ominus} x\right\|^{2}\right) \\
& =\frac{(1-\lambda) \lambda}{2}\left(\frac{1}{(1-\lambda)^{2}} \inf _{k \in K, x-k \in K^{\ominus}}\|k\|^{2}+\frac{1}{\lambda^{2}} \inf _{l \in K^{\ominus}, x-l \in K^{\ominus \ominus}}\|l\|^{2}\right) \\
& \leq \frac{(1-\lambda) \lambda}{2} \inf _{k+l=x, k \in K, l \in K^{\ominus}}\left(\frac{1}{(1-\lambda)^{2}}\|k\|^{2}+\frac{1}{\lambda^{2}}\|l\|^{2}\right) \\
& =\frac{(1-\lambda) \lambda}{2} \inf _{(1-\lambda) u+\lambda v=x, u \in K, v \in K^{\ominus}}\left(\|u\|^{2}+\|v\|^{2}\right) \\
& \leq \frac{(1-\lambda) \lambda}{2} \inf _{(1-\lambda) u+\lambda v=x, u \in K, v \in K^{\ominus}}\left(\|u\|^{2}-2\langle u, v\rangle+\|v\|^{2}\right) \\
& =\frac{(1-\lambda) \lambda}{2} \inf _{(1-\lambda) u+\lambda v=x, u \in K, v \in K^{\ominus}}\|u-v\|^{2} \\
& =f_{\lambda}(x) .
\end{aligned}
$$

Therefore,

$$
f_{\lambda}(x)=\frac{(1-\lambda) \lambda}{2}\left(\frac{1}{(1-\lambda)^{2}}\left\|P_{K} x\right\|^{2}+\frac{1}{\lambda^{2}}\left\|P_{K \ominus} x\right\|^{2}\right)
$$

and the proof of Example 4.9 is complete.

\section{Appendix D. Proof of Theorem 4.11.}

Let us first recall the required notions from convex analysis. Fix $f \in \mathcal{F}$. The subdifferential operator $\partial f$ is the set-valued mapping

$$
x \mapsto\left\{x^{*} \in X \mid(\forall h \in X) f(x)+\left\langle h, x^{*}\right\rangle \leq f(x+h)\right\},
$$

with domain $\operatorname{dom} \partial f:=\{x \in X \mid \partial f(x) \neq \varnothing\}$. Set $q:=\frac{1}{2}\|\cdot\|^{2}$. Note that $\operatorname{ran} \partial(q+$ $f)^{*}=\operatorname{ran}\left((\partial(q+f))^{-1}\right)=\operatorname{dom} \partial(q+f)=\operatorname{dom} \partial f \subseteq \operatorname{dom} f$ and thus $\overline{\operatorname{ran} \partial(q+f)^{*}}=$ $\overline{\operatorname{dom} f}$; see, e.g., [20, Chapters 23 and 24]. The proximal mapping of a function $g \in \mathcal{F}$ is defined by $\partial(q+g)^{*}$; see, e.g., $[18,20]$. Now abbreviate the proximal mapping of $f_{\mu}$ by $P_{\mu}$, for $\mu \in\{0, \lambda, 1\}$. Then [4, Theorem 2.6] states that

$$
P_{\lambda}=(1-\lambda) P_{0}+\lambda P_{1} .
$$

Set $A:=(1-\lambda) P_{0}$ and $B:=\lambda P_{1}$. Observe that $A$ and $B$ are subdifferential operators of functions in $\mathcal{F}$, namely of $(1-\lambda)\left(q+f_{0}\right)^{*}$ and $\lambda\left(q+f_{1}\right)^{*}$, respectively. Thus $A$ and $B$ are maximal monotone (see [20, Corollary 31.5.2] and also [10, 22] for further information), and both satisfy condition $(*)$ of Brézis and Haraux [11]. Since $A+B=P_{\lambda}$ is also maximal monotone, [11, Théorème 3] (see also [22, Section 19]) implies that $\overline{\operatorname{ran}(A+B)}=\overline{\operatorname{ran} A+\operatorname{ran} B}$ and $\operatorname{int}(\operatorname{ran}(A+B))=\operatorname{int}(\operatorname{ran} A+$ $\underline{\operatorname{ran} B) \text {. Hence } \overline{\operatorname{dom} f_{\lambda}}}=\overline{\operatorname{ran} \partial\left(q+f_{\lambda}\right)^{*}}=\overline{\operatorname{ran} P_{\lambda}}=\overline{\operatorname{ran}(A+B)}=\overline{\operatorname{ran} A+\operatorname{ran} B}=$ $\overline{(1-\lambda) \operatorname{ran} P_{0}+\lambda \operatorname{ran} P_{1}}=\overline{(1-\lambda) \operatorname{dom} \partial f_{0}+\lambda \underline{\operatorname{dom} \partial f_{1}}}=\overline{(1-\lambda) \operatorname{dom} f_{0}+\lambda \operatorname{dom} f_{1}}$. Consequently, $\operatorname{int} \operatorname{dom} f_{\lambda}=\operatorname{int} \overline{\operatorname{dom} f_{\lambda}}=\operatorname{int} \overline{(1-\lambda) \operatorname{dom} f_{0}+\lambda \operatorname{dom} f_{1}}=\operatorname{int}((1-$ $\lambda) \operatorname{dom} f_{0}+\lambda \operatorname{dom} f_{1}$ ). This completes the proof of Theorem 4.11 . 
Acknowledgments. We wish to thank Roger Wets and Jon Vanderwerff for helpful discussions concerning the material in Sections 5 and 6 , respectively. We also wish to thank two anonymous referees and the Section Editor Dr. A. Bernoff for numerous pertinent comments and suggestions.

\section{REFERENCES}

[1] H. Aтtouch, Variational Convergence for Functions and Operators, Applicable Mathematics Series, Pitman (Advanced Publishing Program), Boston, MA, 1984.

[2] H. Attouch and R. J.-B. Wets, Isometries for the Legendre-Fenchel transform, Trans. Amer. Math. Soc., 296 (1986), pp. 33-60.

[3] H. H. Bauschke, Duality for Bregman projections onto translated cones and affine subspaces, J. Approx. Theory, 121 (2003), pp. 1-12.

[4] H. H. Bauschke, E. Matoušková, And S. Reich, Projection and proximal point methods: Convergence results and counterexamples, Nonlinear Anal., 56 (2004), pp. 715-738.

[5] H. H. Bauschke and M. von Mohrenschildt, Symbolic computation of Fenchel conjugates, SIGSAM Bull., (2006).

[6] G. Beer, Topologies on Closed and Closed Convex Sets, vol. 268 of Mathematics and its Applications, Kluwer Academic Publishers Group, Dordrecht, 1993.

[7] J. M. Borwein And C. H. Hamilton, Symbolic convex analysis: algorithms and examples. Math. Prog., to appear.

[8] J. M. Borwein And A. S. Lewis, Convex Analysis and Nonlinear Optimization, CMS Books in Mathematics/Ouvrages de Mathématiques de la SMC, 3, Springer-Verlag, New York, 2000. Theory and examples.

[9] S. Boyd and L. Vandenberghe, Convex Optimization, Cambridge University Press, Cambridge, 2004.

[10] H. BRÉZIS, Opérateurs Maximaux Monotones et Semi-groupes de Contractions dans les Espaces de Hilbert, North-Holland Publishing Co., Amsterdam, 1973. North-Holland Mathematics Studies, No. 5. Notas de Matemática (50).

[11] H. BrÉzis AND A. HARAux, Image d'une somme d'opérateurs monotones et applications, Israel J. Math., 23 (1976), pp. 165-186.

[12] C. H. Hamilton, Symbolic convex analysis, Master's thesis, Simon Fraser University, 2005.

[13] J.-B. Hiriart-Urruty and C. Lemaréchal, Convex Analysis and Minimization Algorithms, vol. 305-306 of Grundlehren der Mathematischen Wissenschaften [Fundamental Principles of Mathematical Sciences], Springer-Verlag, Berlin, 1993. Vol I: Fundamentals, Vol II: Advanced theory and bundle methods.

[14] A. J. JARvis, A brief manual to using Lucet's code, tech. report, University of Guelph, June 2004. Manuscript prepared for an Undergraduate Summer Research Project.

[15] R. Lucchetti, Convexity and Well-posed Problems, CMS Books in Mathematics/Ouvrages de Mathématiques de la SMC, 22, Springer, New York, 2006.

[16] Y. LUCET, Fast Moreau envelope computation I: Numerical algorithms, Numerical Algorithms, (2007). DOI - 10.1007/s11075-006-9056-0.

[17] Y. Lucet, H. H. BAuschke, And M. TRIEnis, The piecewise linear-quadratic model for computational convex analysis, tech. report, University of British Columbia, July 2006.

[18] J.-J. Moreau, Proximité et dualité dans un espace Hilbertien, Bull. Soc. Math. France, 93 (1965), pp. 273-299.

[19] J.-P. PenOt AND C. ZăLINESCU, Continuity of usual operations and variational convergences, Set-Valued Anal., 11 (2003), pp. 225-256.

[20] R. T. Rockafellar, Convex Analysis, Princeton University Press, Princeton, New york, 1970.

[21] R. T. Rockafellar and R. J.-B. Wets, Variational Analysis, Springer-Verlag, Berlin, 1998.

[22] S. Simons, Minimax and Monotonicity, vol. 1693 of Lecture Notes in Mathematics, SpringerVerlag, Berlin, 1998.

[23] C. ZăLinescu, Convex Analysis in General Vector Spaces, World Scientific Publishing Co. Inc., River Edge, NJ, 2002. 